Accepted pre-print version of :

Nees, M.A., Sharma, N., \& Herwig, K. (in press). Some characteristics of mental models of advanced driver assistance systems: A structured interviews approach. Accepted for publication in Proceedings of the Human Factors and Ergonomics Society 64th Annu Meeting. To be presented October, 2020.

\title{
Some Characteristics of Mental Models of Advanced Driver Assistance Systems: A Semi-structured Interviews Approach
}

\author{
Michael A. Nees, Nithya Sharma, and Karli Herwig \\ Lafayette College
}

\begin{abstract}
People construct mental models - internal cognitive representations-when they interact with dynamic systems. The introduction of automation in vehicles has raised concerns about potential negative consequences of inaccurate mental models, yet characteristics of mental models remain difficult to identify. A descriptive study used semi-structured interviews to explore mental models of advanced driver assistance systems (adaptive cruise control, lane keeping assist, and Level 2 systems). Results exposed shortcomings in drivers' understandings of the hardware, software, and limitations of these systems and also suggested that mental models will affect behavior while using automation. Further, we found that mental models can be influenced by interface feedback (or lack thereof) and limitations experienced. Some drivers attributed purposeful design to aspects of the systems that likely were chosen idiosyncratically or arbitrarily. Our findings offered potentially useful avenues for future research on mental models of automation and corroborated concerns that inaccurate mental models may be common.
\end{abstract}

\section{INTRODUCTION}

Cognitive scientists long have held that, when interacting with a dynamic system, people construct a mental model - an internal representations of the system. Mental models entail descriptions and explanations of functions as well as predictions about how the system will work in the future (see, e.g., Rouse \& Morris, 1986). These descriptions, explanations, and predictions may be inaccurate, however; Norman (1983) described mental models as "incomplete," "unstable," "unscientific," "superstitious," and "parsimonious."

Accurate mental models have been indicated as important for effective interactions with dynamic systems, particularly when the system automates complex tasks or processes (Endsley, 1996). The deployment of technology to automate driving tasks, for example, has raised concerns about the human driver's ability to develop accurate mental models of the automation (e.g., Endsley, 2019). Specifically, recent perspectives have theorized that mental models of vehicle automation influence drivers' trust and situation awareness (Heikoop et al., 2016), and inaccurate mental models may foster trust in vehicle automation in driving scenarios in which trust is not warranted (e.g., Kazi et al., 2007).

Inaccurate mental models appear to be a problem with advanced driver assistance systems (ADAS) that are currently available to consumers. For example, a cluster analysis (Dickie \& Boyle, 2009) suggested that drivers can be grouped as aware, unsure, or unaware of adaptive cruise control (ACC) limitations. Unsure and unaware users were more likely to engage the system in potentially problematic roadway conditions. All three groups reported high trust in the system, though unaware or unsure users apparently may trust ACC in situations in which they should not. Seppelt and Lee, (2015) used computational modeling to demonstrate that inaccurate mental models are likely to result in collisions in fairly common situations during which driver intervention is required to overcome ACC limitations. Under a reasonable set of assumptions, their study showed that responses to perceptual warnings alone would not result in satisfactory safety margins in the absence of an accurate understanding of system functionality and limitations.

Despite a strong consensus among vehicle automation researchers regarding the importance of mental models, the study of mental models presents difficulties for measurement. Rouse and Morris (1986) discussed the challenges of differentiating "mental models" from simply "knowledge" (i.e., of how a system functions). Although a complete treatment of this topic is beyond the scope of the current discussion, it is probably fair to conclude that accurate declarative knowledge of how a system functions is likely necessary, but also insufficient and incomplete, evidence of an accurate mental model.

Still, in the absence of a standardized measurement approach (and given the high cost of qualitative approaches such as interviews, see Morgan et al., 2002), researchers to date have measured mental models of ADAS using scores on knowledge-based surveys and questionnaires (see Beggiato \& Krems, 2013; Seppelt \& Lee, 2019; Stanton \& Young, 2005). This approach requires that the breadth of information relevant to the mental model (and its accompanying vocabulary among users) is known to the researcher who designs the instrument (Morgan et al., 2002).

Fewer studies have assessed the qualitative content of mental models of driver assistance systems (but see Jenness et al., 2019; Strand et al., 2018). Although qualitative research approaches are resource-intensive and less amenable to easy quantification, they can help to establish the range of beliefs that compose mental models, as well as the vocabulary used by people to describe those beliefs (Morgan et al., 2002).

The current study used qualitative, semi-structured interviews to explore mental models of ADAS. We interviewed drivers who have used SAE Level 1 or 2 systems (SAE On-road Automated Vehicles Standards Committee, 2018) including ACC, lane keeping assist (LKA), and Level 2 systems (with simultaneous ACC and lane centering steering). 


\section{Participants}

\section{METHOD}

Participants $(N=25 ; 17$ females, 8 males; $M$ age $=$ 27.71, $S D=15.20$, range $=19-71$, four ages missing) with direct experience using Adaptive Cruise Control, Lane Keeping Assist, or a Level 2 automated system were recruited from Lafayette College. Participants were required to have experience with at least one of these systems to be eligible for this study. Participants were queried twice (via email before arriving to the interview and at the beginning of the interview) to re-affirm eligibility. Participants were recruited from a campus-wide email and an online recruitment system. Twenty participants identified as undergraduate students while the remaining five participants were faculty or staff. Participants were compensated for their participation with a choice of 10 dollars or course extra credit. Three participants declined compensation. Participants reported $M=10.16(S D=13.79)$ years of driving experience (range $=2-55$ years) and reported driving from zero to 22.5 hours per week. Participants' most frequent driving environments included rural/small town driving $(n=14)$, distance/interstate/ freeway driving $(n=10)$ and urban/city driving $(n=2)$; one participant made more than one selection.

Participants reported using ACC $(n=14)$, LKA $(n=18)$, and Level 2 systems (that perform ACC with lane centering steering simultaneously) $(n=5)$. Vehicle makes included Subaru $(n=5)$, Toyota $(n=4)$, Honda $(n=4)$, Tesla $(n=2)$, Lexus $(n=2)$, and 1 each of Mercedes, Mazda, Infiniti, Genesis, Audi, Nissan, BMW, and Buick. Experience with and frequency of use of ADAS systems was highly variable. Some participants reported years of experience or daily use of ADAS, whereas others had only used ADAS 1-2 times. Participants reported learning to use the systems by trial and error $(n=17)$, with help from a friend or family member who knew how to use the system $(n=9)$, by reading the instruction manual $(n=2)$, and by consulting other (presumably online) information $(n=1)$.

\section{Interview Questions and Procedure}

The questions that guided the semi-structured interviews are provided in the Appendix. A given participant did not necessarily answer every question. Instead, the interview proceeded in a conversational manner; participants were encouraged to share as much as possible with as little prompting from the researchers as possible. We did not want to induce demand characteristics in participants. Thus, the interviewers flexibly used a selection of the questions and probes to move the conversation forward. Since participants with sparse mental models may have felt compelled to invent a mental model on the spot under questioning, the interviewers were careful to avoid suggestive or repetitive questioning. This approach contributed to variability in the amount of information provided by each participant (see below).

The study was approved by the Lafayette College IRB. After signing an informed consent, participants were interviewed by two researchers (the second and third authors). One researcher led the interview, while the other took notes on a data collection sheet and asked follow-up questions on an ad hoc basis. Interviews were 30-45 minutes in duration. Interviews were not recorded.

\section{RESULTS}

Following each interview, the two interviewers immediately coded the most relevant information from the interview into statements expressing beliefs held by participants about driver assistance systems. A consensus agreement approach was used. Consensus approaches to coding qualitative data use rigorous discussion among the researchers (rather than quantification of reliability) to arrive at classifications (Harry et al., 2005; Richards \& Hemphill, 2018). During coding, beliefs were represented in the general form "my car does X because Y." The researchers translated their interview notes into as many belief statements as they could extract. The researchers were able to identify an average of 4.88 beliefs ( $\mathrm{SD}=2.57$, range $=1$ to 13 ) per participant. Later, another researcher (the first author) translated the belief statements into the quantities reported below; both the coded belief statements and the original interview notes were consulted as needed.

\section{Hardware and Software Beliefs}

Most $(n=22)$ participants explicitly indicated (in a general sense) that the ADAS in their vehicle relied on sensing hardware to negotiate driving scenarios. Most indicated that the sensing hardware included a camera $(n=16)$, although two participants explicitly indicated that their vehicle did not use cameras. One of those participants owned a vehicle whose manufacturer's website indicates the vehicle has eight cameras. Beyond cameras, there was variability in the types of sensors participants indicated were part of sensing hardware. Participants reported that their vehicles use radar $(n=5)$, sonar $(n=3)$, color sensors $(n=2)$, and one instance each of pressure sensors in the steering wheel, tire rotation sensors (to detect distance traveled), heat/temperature sensors (to detect objects), long and short distance sensors (to detect near and far objects), metallic sensors (to detect paint in lane lines), motion sensors, and a sensor to detect the composition of materials (to differentiate road dividers from obstacles). Four participants explicitly indicated that different ADAS systems (e.g., ACC and LKA) in their vehicle use the same sensors, whereas five participants indicated that different systems use different sensors. Five participants indicated that their ADAS systems use GPS; one person explicitly indicated that their system does not use GPS.

Participants often stated where physically on the car they believe sensors are located, including general statements that indicated multiple sensors are located around the car and/or distributed in different parts of the car $(n=4)$. Other locations mentioned included on the side of the car $(n=7)$, on the front of the car $(n=5)$, on the steering wheel (for driver monitoring) $(n=3)$, on the back or rear of the car $(n=3)$, on the bottom of the car $(n=2)$, on the roof of the car $(n=1)$, on the brake 
pedal $(n=1)$, on the gas pedal $(n=1)$, near the window $(n=$ $1)$, and in the tires $(n=1)$.

Five participants made statements indicating that their system uses a central computer or software, and three participants made statements indicating a belief that their system does not use a central computer. One participant mentioned AI (artificial intelligence) as a characteristic of the software, and one participant explicitly mentioned algorithms. Two participants described a specific algorithm; both believed that the camera system uses changes in the size of objects from frame-to-frame (looming, etc.) to judge distance.

\section{Human-machine Interface Beliefs}

Participants who mentioned the HMI noted auditory alerts $(n=11)$, visual indicators $(n=7)$, and vibrations or haptic feedback $(n=4)$. Four participants described the HMI alerts as annoying. The design of the HMI appears to influence mental models; participants $(n=7)$ made statements indicating that they inferred functions and capabilities of the system from the HMI feedback. An equal number $(n=7)$ indicated that they had inferred functions and capabilities of the system from the absence or lack of HMI feedback in some scenarios.

\section{Beliefs about Limitations and Perceived Reliability}

Participants stated beliefs indicating a number of different conditions or circumstances in which their ADAS systems are limited. Participants most commonly mentioned speed thresholds for engaging systems $(n=7)$. Participants had different beliefs about why the systems implemented speed thresholds. For example, two participants indicated that lack of ACC functionality at low speeds was intended to promote driver attention and awareness at low speeds; those participants believed low speeds objectively entail more potential hazards. Three participants indicated that LKA turns on at higher speeds (e.g., $>30 \mathrm{mph}$ ), because the potential consequences of lane departures are greater at higher speeds. The aforementioned mental models, therefore, ascribe safetyminded, intentional design to the limitations encountered. Other participants believed speed thresholds were necessitated by technological limitations. Two participants indicated that LKA speed thresholds were designed to attenuate false alarms (beeping) in low speed scenarios involving obstacles (parking lots, etc.). One participant believed there are too many variables to safely operate $\mathrm{ACC}$ at low speeds.

Additional limiting circumstances noted by participants included limitations in sensing obstacles $(n=2)$, in low light or at night $(n=2)$, and when the camera is dirty $(n=2)$. Some participants noted that the system doesn't recognize traffic lights or signs $(n=2)$, and that the system works better on highways than other types of roads $(n=2)$. Participants noted that LKA is limited by ambiguous or poor lane-markings ( $n=$ 2). Other limiting conditions mentioned once included sloping roadways, construction zones, and scenarios that require hard braking. Three participants reported experiencing false negatives with ACC; one person complained explicitly of false positives with LKA.
In most interviews, participants were asked to rate the perceived reliability of their ADAS on a scale from 0 (never works the way it is supposed to work) to 100 (always works the way it is supposed to work). ACC users $(n=12)$ who provided scores rated the technology's reliability as $M=89 \%$, range $=60$ to 100 , with two participants rating ACC as $100 \%$ reliable. LKA users $(n=17)$ who provided scores rated the technology's reliability as $M=82 \%$, range $=30$ to 100 , with three participants rating LKA as $100 \%$ reliable. For Level 2 ADAS, participants $(n=5)$ who provided scores on average rated the technology's reliability as $M=88 \%$, range $=75$ to 95 , with no participants rating the systems as $100 \%$ reliable. During interviews, several participants indicated that they rated the systems as less than $100 \%$ reliable simply because it felt wrong to choose $100 \%$; these participants did not feel the technology was unreliable.

One participant believed that lane keeping assist is more effective on busy roadways and curved roads. They believed that the system was reliable and more precise and consistent than a human driver in those circumstances. In truth, busier roadways almost certainly present more opportunities for collisions and system errors. A second participant also believed that LKA performed especially well on curved roads. They indicated that curved roads involve more demanding lane-keeping, so they seemed to infer that the system was designed to be especially useful in more difficult lane-keeping scenarios. The same participant also interpreted the greater frequency of feedback (alerts) from LKA on curved roads to indicate that it was working more effectively. Most owner's manuals indicate curved roadways as a scenario in which systems like ACC and LKA are potentially more limited - not more effective.

\section{Beliefs about Safety}

One participant indicated that they believe they can pay less attention to the driving task because of the extra safety afforded by ADAS; researchers have expressed concerns about these types of behavioral adaptations to driver assistance systems (Rudin-Brown \& Noy, 2002). Similarly, one participant indicated that they tended to consume more foods that require eating with spoons while driving using ADAS. Another participant felt that ADAS made them less aware of cars coming in to their driving lane, yet also felt like they must pay more attention to monitoring the ADAS systems. Two participants described the ADAS as sensitive or too sensitive; one of those participants described driving more carefully to avoid hearing alerts. Six participants made statements indicating that they have concerns about becoming overreliant on ADAS systems.

Two participants explicitly mentioned feeling safer with ADAS; one participant explicitly mentioned feeling less safe. At least two participants indicated that they worry about other drivers misusing ADAS technology; these participants were both non-students (and thus were among the more experienced drivers in the sample). Similarly, one participant reported that the system is $75 \%$ reliable overall but always safe when they are the driver. 


\section{DISCUSSION}

\section{Strengths, Limitations, and Caveats}

Mental models are incomplete, individualized, unscientific, and superstitious (Norman, 1983). By their nature, mental models can be difficult to anticipate and discern from known expert/conceptual models of systems. As such, open-ended, qualitative approaches like the interviews used here allow participants the opportunity to express the variability of beliefs underlying mental models in their own words. Qualitative research can serve as an important precursor to the development of standardized, efficient, quantitative measures of mental models (for a review, see Morgan et al., 2002).

Although the sampling procedure used here does not permit broad generalizations, the strength of the approach taken in the current study is likely in the variability of participants' responses rather than the central tendency. Thus, we believe that the presence of a given belief among even a small number of the participants sampled here could represent an important aspect of mental models and/or a marker for future investigations, but the absence any particular piece of information is perhaps more ambiguous. In interpreting these results, omissions could reflect the absence of information in a mental model, but participants also might have neglected to mention parts of their mental models during the interview for any number of reasons, including failures of memory and/or lack of explicit access to implicit knowledge.

The sample size in the current study was small. The drivers interviewed here were mostly female and mostly young adults. They also represented primarily college students from only one geographic region. Our drivers' amount of experience with and frequency of use of ADAS ranged a great deal, which precluded interpretations regarding how experience impacts mental models. To an extent, the analyses reported here also confound different types of ADAS (ACC, LKA, and Level 2 systems), although this decision seems reasonable give that these technologies increasingly co-occur in newer vehicles.

Given these limitations, this research should be viewed as descriptive, exploratory, and suggestive of the scope of beliefs that might compose mental models of ADAS. The conclusions presented here are tentative and should be viewed as one piece of evidence in emerging understandings of mental models of ADAS.

\section{Conclusions: Some Characteristics of Mental Models of ADAS}

Drivers' mental models of ADAS are variable in both completeness and content. Drivers have different background knowledge and interest in driver assistance systems (Strand et al., 2018). Our interviews suggested that some drivers possess very sparse mental models of ADAS (also see Hollearn \& Miles, 2019), whereas others have developed more extensive mental models. Future research should examine whether more shallow mental models are associated with lack of experience or instead represent some other individual difference (e.g., lack of interest or curiosity).

Drivers vary in their understandings of (and some have inaccurate beliefs about) ADAS hardware and software. Although most participants indicated explicit awareness of ADAS's use of sensory hardware, especially cameras, some mental models involved incorrect understandings of the vehicle's sensory apparatuses Our interviews suggested that beliefs about computing and data processing are less prominent in mental models of ADAS than beliefs about sensory hardware, perhaps because data processing elements are more abstract and opaque to the user. More research is needed to understand how inaccuracies in drivers' mental models of hardware and software might affect behavior, but it seems reasonable to expect that misunderstandings may have downstream effects on drivers' interactions with ADAS.

The design of the HMI can influence the formation of mental models of ADAS (for better or worse). The ACC feedback provided by the HMI varies across manufacturers (Wright et al., 2019). In our interviews, some drivers formed impressions of system functionality based on the design of visual displays in the HMI. For example, a driver inferred that different sensors were used for driving versus parking, because the visual icon in the HMI was different during these activities. Several drivers also reported making inferences about the vehicle's functionality based on the lack of HMI feedback in certain driving scenarios. Since drivers seem to use the HMI to develop mental models, care should be taken to design HMIs that promote accurate understandings of the system. Seppelt and Lee, (2007), for example, showed that displays that promote transparency regarding system functions (limitations, etc.) resulted in safer driving behaviors. Arbitrary and/or idiosyncratic display choices that do not map tightly and intuitively to system functionality may contribute to the development of inaccurate mental models.

System limitations are part of mental models, but drivers' understanding of limitations of ADAS appears to be incomplete. Participants commonly reported awareness of one or more circumstances that limit the functionality of ADAS systems. Still, the frequency and variety of limitations in drivers' mental models perhaps did not reflect the scope of limitations noted in expert/conceptual models of ADAS systems (derived from limitations commonly listed in owners' manuals, etc.). Drivers tended to perceive that the reliability of ADAS systems is high. Although this perception perhaps is accurate, a few participants perceived either ACC or LKA to be $100 \%$ reliable, which could lead to potentially problematic behaviors such as overreliance.

Drivers may interpret system limitations that are incidental and technical to be purposeful design decisions. We noted potentially important differences in how drivers interpreted the limitations they encountered in ADAS systems. Some drivers seem to ascribe limitations to the intentions of engineers/designers; they believed that system limitations were deliberately included to promote safety or some other rational goal. In other instances, drivers attributed limitations to the fallibility of the technology underlying the system. Either interpretation could be accurate, depending on the particular circumstance. Still, engineers and designers should 
be aware of the potential for incidental technical limitations in ADAS to be interpreted by drivers as purposeful or included in the service of accomplishing a goal (related to safety, etc.).

Mental models may contribute to behavioral adaptation. Some aspects of participants' mental models appeared to be linked to behavioral changes while using ADAS systems. A few participants reported changing driving habits in ways that might be less safe (e.g., paying less attention, eating with spoons while driving), while others reported changing driving behaviors in ways that might enhance safety (e.g., driving more carefully to avoid ADAS alerts). Several participants were concerned with becoming overreliant on ADAS, which suggested that they do not fully trust the systems. Interestingly, some participants expressed concern over potential behavioral adaptations of other drivers, which likely represents an extension of well-documented better-thanaverage effects (for a review, see Nees, 2019).

Inaccurate mental models of automation remain a concern. A longstanding concern is that inaccurate mental models foster inappropriate, unsafe behaviors when interacting with automation. We noted several instances of erroneous beliefs that seemed likely to lead to unsafe behaviors. The most notable examples included participants who believed ADAS actually was more effective in driving scenarios during which ADAS is, in fact, less effective and should not be used.

\section{REFERENCES}

Beggiato, M., \& Krems, J. F. (2013). The evolution of mental model, trust and acceptance of adaptive cruise control in relation to initial information. Transportation Research Part F: Traffic Psychology and Behaviour, 18, 47-57. https://doi.org/10.1016/j.trf.2012.12.006

Dickie, D. A., \& Boyle, L. N. (2009). Drivers' understanding of adaptive cruise control limitations. Proceedings of the Human Factors and Ergonomics Society Annual Meeting, 53, 1806-1810.

Endsley, M. R. (1996). Automation and situation awareness. Automation and Human Performance: Theory and Applications, 20, 163-181.

Endsley, M. R. (2019). Human-automation interaction and the challenge of maintaining situation awareness in future autonomous vehicles. In M. Mouloua \& P. A. Hancock (Eds.), Human Performance in Automated and Autonomous Systems: Current Theory and Methods. CRC Press.

Harry, B., Sturges, K. M., \& Klingner, J. K. (2005). Mapping the process: An exemplar of process and challenge in grounded theory analysis. Educational Researcher, 34(2), 3-13.

Heikoop, D. D., de Winter, J. C., van Arem, B., \& Stanton, N. A. (2016). Psychological constructs in driving automation: A consensus model and critical comment on construct proliferation. Theoretical Issues in Ergonomics Science, 17(3), 284-303.

Hollearn, M. K., \& Miles, J. D. (2019). Do People Mentally Represent Automated Tasks? Evidence from Task-Switching Costs Following Takeovers. Proceedings of the Human Factors and Ergonomics Society Annual Meeting, 63, 1227-1231.

Jenness, J., Lenneman, J., Benedick, A., Huey, R., Jaffe, J., Singer, J., \& Yahoodik, S. (2019). Spaceship, Guardian, Coach: Drivers' Mental Models of Advanced Vehicle Technology. International Conference on Human-Computer Interaction, 351-356.

Kazi, T., Stanton, N. A., Walker, G. H., \& Young, M. S. (2007). Designer driving: Drivers' conceptual models and level of trust in adaptive cruise control. International Journal of Vehicle Design, 35(3), 339-360.

Morgan, M. G., Fischhoff, B., Bostrom, A., \& Atman, C. J. (2002). Risk Communication: A Mental Models Approach. Cambridge University Press.
Nees, M. A. (2019). Safer than the average human driver (who is less safe than me)? An examination of a popular safety benchmark for selfdriving cars. Journal of Safety Research, 69, 61-68.

Norman, D. A. (1983). Some observations on mental models. In D. Gentner \& A. L. Stevens, (Eds.), Mental Models (pp. 7-14). Psychology Press.

Richards, K. A. R., \& Hemphill, M. A. (2018). A practical guide to collaborative qualitative data analysis. Journal of Teaching in Physical Education, 37(2), 225-231.

Rouse, W. B., \& Morris, N. M. (1986). On looking into the black box: Prospects and limits in the search for mental models. Psychological Bulletin, 100(3), 349.

Rudin-Brown, C. M., \& Noy, Y. I. (2002). Investigation of behavioral adaptation to lane departure warnings. Transportation Research Record, 1803(1), 30-37.

SAE On-road Automated Vehicles Standards Committee. (2018). SAE J3016: Taxonomy and Definitions for Terms Related to On-Road Motor Vehicle Automated Driving Systems (SAE J3016; pp. 1-35).

Seppelt, B. D., \& Lee, J. D. (2007). Making adaptive cruise control (ACC) limits visible. International Journal of Human-Computer Studies, 65(3), 192-205.

Seppelt, B. D., \& Lee, J. D. (2015). Modeling driver response to imperfect vehicle control automation. Procedia Manufacturing, 3, 26212628.

Seppelt, B. D., \& Lee, J. D. (2019). Keeping the driver in the loop: Dynamic feedback to support appropriate use of imperfect vehicle control automation. International Journal of Human-Computer Studies, 125, 66-80. https://doi.org/10.1016/j.ijhcs.2018.12.009

Stanton, N. A., \& Young, M. S. (2005). Driver behaviour with adaptive cruise control. Ergonomics, 48(10), 1294-1313.

Strand, N., Stave, C., \& Ihlström, J. (2018). A case-study on drivers' mental model of partial driving automation. 25th ITS World Congress, Copenhagen, Denmark, 17-21 September 2018.

Wright, T. J., Svancara, A. M., \& Horrey, W. J. (2019). Consumer Information Potpourri: Instructional and Operational Variability Among Passenger Vehicle Automated Systems. Ergonomics in Design, 28(1), 4-15.

\section{APPENDIX \\ Semi-structured interview questions}

1. What advanced driver assistance systems do you have in your car? 2. Please tell me everything you know about the [adaptive cruise control/lane keeping/etc.] system in your car. Prompts: What does it do? (Functionality) How does it work? (Probe understanding of parts, engineering etc.) When do you use it? Where do you use? How often do you use it? For how long have you used it? How do you know if it's on/off/ working? (Probe HMI; sounds, lights, feedback etc.) If you had to teach someone who has never used it before, can you walk me through what you would say to them to show them how to use it?3. Have there been any times when the system did something that you did not expect? Something that was surprising to you? 4 . How did you learn to use the system?

5. How do your driving habits change while using these features? 6. How does using the system affects safety while driving? 7. How reliable is the system in your car? If 100 means it always works the way it is supposed to work, and 0 means it never works the way it is supposed to work, how would you rate your system from 0 to 100 ? Why did you choose this rating--can you give me some examples? 